\title{
Clinical significance and prognostic value of Forkhead box A1 expression in human epithelial ovarian cancer
}

\author{
KAI WANG $^{1 *}$, CHENAN GUAN $^{2 *}$, CHENYAN FANG $^{3 *}$, XIAOXIAO JIN $^{1}$, \\ JUNHUI YU ${ }^{1}$, YUQUAN ZHANG ${ }^{4}$ and LINGZHI ZHENG ${ }^{1}$
}

\begin{abstract}
Departments of ${ }^{1}$ Obstetrics and Gynecology and ${ }^{2}$ Kidney Internal Medicine, Taizhou Hospital of Zhejiang Province, Wenzhou Medical University, Linhai, Zhejiang 317000; ${ }^{3}$ The Second Clinical Medical College, Zhejiang Chinese Medical University, Hangzhou, Zhejiang 310053; ${ }^{4}$ Department of Obstetrics and Gynecology, Affiliated Hospital of Nantong University, Nantong, Jiangsu 226001, P.R. China
\end{abstract}

Received August 12, 2016; Accepted July 3, 2017

DOI: 10.3892/ol.2018.7899

\begin{abstract}
Forkhead box (FOX) A1 is a member of the FOX family of transcription factors, which serve a function in numerous types of tumor. The present study assessed the potential role of FOXA1 in human epithelial ovarian carcinoma (EOC). Total RNA was isolated from 16 fresh-frozen EOC tumors with paired corresponding non-malignant ovarian epithelium tissues, and FOXA1 expression was analyzed using reverse transcription-quantitative polymerase chain reaction. Immunohistochemical analysis was performed to evaluate FOXA1 expression in 110 epithelial ovarian carcinoma tissue specimens (including 80 serous papillary adenocarcinoma, 9 clear cell carcinoma, 12 endometrioid adenocarcinoma, 5 mucinous carcinoma and 4 transitional cell carcinoma specimens), 24 benign ovarian tumor surface epithelium tissues and 10 normal ovarian tissue samples. The present study analyzed the association between FOXA1 expression and clinical characteristics in patients with EOC. The Kaplan-Meier method was used for survival analysis. The results of the present study revealed that FOXA1 mRNA expression was significantly increased in EOC tissues compared with paired normal ovarian samples $(\mathrm{P}=0.014)$. The immunohistochemical expression of FOXA1 in EOC tissues was associated with the FIGO grade, differentiation
\end{abstract}

Correspondence to: Dr Yuquan Zhang, Department of Obstetrics and Gynecology, Affiliated Hospital of Nantong University, 19 Xisi Road, Nantong, Jiangsu 226001, P.R. China

E-mail: m13362627683@163.com

Dr Lingzhi Zheng, Department of Obstetrics and Gynecology, Taizhou Hospital of Zhejiang Province, Wenzhou Medical University, 150 Ximen Road, Linhai, Zhejiang 317000, P.R. China E-mail: zhenglz123@126.com

\section{*Contributed equally}

Key words: Forkhead box A1, expression, prognostic value, clinical significance, epithelial ovarian cancer status and overall survival time (all $\mathrm{P}<0.05)$. Finally, the significance of FOXA1 expression in the prognosis of the patients was evaluated. The results of Kaplan-Meier survival curve revealed that high FOXA1 expression was associated with decreased overall survival time in the patients, relative to low FOXA1 expression ( $\mathrm{P}=0.0132)$. In conclusion, FOXA1 is overexpressed in EOC and associated with clinicopathological features, including overall survival time. FOXA1 potentially represents a novel biomarker and therapeutic target for EOC.

\section{Introduction}

Epithelial ovarian cancer (EOC) is associated with the second highest incidence and highest mortality rate among gynecological malignancies worldwide. At present, the survival rate remains poor for patients with advanced EOC $(1,2)$. There were 21,880 new cases of EOC diagnosed and 13,850 mortalities in the United States in 2010 (3). The poor prognosis of EOC is primarily due to its advanced stage at the time of diagnosis (4). EOC mortality occurs predominantly due to metastasis; without effective screening tests or the appearance early symptoms, the majority of patients with EOC are diagnosed with metastatic disease (2). Approximately $75 \%$ of patients are diagnosed with advanced (stage III/IV) ovarian carcinoma, which is characterized by peritoneal or distant metastases, respectively, and for which the 5-year survival rate is $15-20 \%$; the rate for patients diagnosed during the early stage (stage I/II) is $80-90 \%$ (5). The majority of cases may be curable if the disease is diagnosed at the early stage. However, the molecular mechanism of EOC aggressiveness has yet to be fully characterized. At present, there are no reliable and accurate markers that predict aggressive phenotypes. Therefore, establishing novel approaches to increase the sensitivity and decrease resistanceto EOC therapy remains critical.

Forkhead box (FOX) A1 representsa potential candidate gene for therapeutic targeting in human EOC; FOXA1 is a transcription factorthat is expressed widely and functions in the development of numerous types of human tissue $(6,7)$. FOXA1, also known as hepatocyte nuclear factor $3 \alpha$, belongs to a superfamily of winged helix transcription factors and has been 
identified as a hepatocyte-enriched transcription factor required for the expression of transthyretin and $\alpha$-1-antitrypsin (8-10). FOXA1 has attracted attentionas it interacts with cis-regulatory regions in heterochromatin to enhance the interaction of ERa with chromatin (11). FOXA1 also serves important functions during multiple phases of mammalian life, including in the regulation of the development of the endodermal layer andorganogenesis, as well as in metabolism and homeostasis in the adult (12-14). FOXA1 is an endodermal 'pioneer transcription factor', which bindsto the promoters and enhancersto enable chromatin access to other tissue-specific transcription factors $(15,16)$. FOXA1 is highly expressed in lung tissue (6). Previous studies have revealed that FOXA1 expression is increased in liver, colon, thyroid and esophageal cancer (17-19). FOXA1 is also essential for the estrogen signaling pathway in breast cancer cells, which is associated with a favorable prognosis (20). It has been suggested that FOXA1 influences androgen receptor (AR) binding to chromatinin androgen-dependent and androgen-independent prostate cancer $(21,22)$. However, no studies have reported on the function of FOXA1 in epithelial ovarian tumors to the best of our knowledge.

To the best of our knowledge, there have also been no studies published on the association between FOXA1 expression and clinical features to determine its clinicopathological significance in human EOC. Therefore, the present study assessed whether the expression of FOXA1 may serve as a novel biomarker for the prognosis of patients with OC and whether it may be suitable for development as a target for therapy. FOXA1 gene expression in EOC samples was compared with benign ovarian tumor surface epithelia and normal ovarian tissues. The association between FOXA1 expression and clinicopathological data in a group of patients with EOC was also analyzed. Finally, the prognostic potential of FOXA1 protein expression in EOC was evaluated.

\section{Materials and methods}

Pathological samples. Formalin-fixed, paraffin-embedded ovarian tissues were harvested from 110 cases of primary epithelial ovarian carcinoma and 24 benign ovarian tumor surface epithelium samples. The patients with ovarian tumors underwent surgery from January 2003 to December 2007 at the Affiliated Hospital of Nantong University (Nantong, China). The 10 healthy individual ovary samples were also harvested for comparison. The clinical stage of all the patients with EOC was determined using the FIGO staging system (23); 64 cases were low stage tumors (I-II) and 46 were high stage tumors (III-IV). Neither preoperative radiation nor chemotherapy had been received by the patients with EOC. Clinical information on each patient, including age, histological type, grade based on the World Health Organization (WHO) criteria (24), FIGO stage and tumor size, was collected from medical records, including from a 5-year follow-up. The mean age of the patients was 54.5 years (range, 29-78 years). The protocol of the present study was approved by the Institutional Review Board at the Affiliated Hospital of Nantong University. Informed written consent was obtained for each patient.

Reverse transcription-quantitative polymerase chain reaction $(R T-q P C R)$. EOC fresh-frozen at $-80^{\circ} \mathrm{C}$ and paired normal ovarian epithelium samples $(\mathrm{n}=16$ of each) were obtained from January 2011 to August 2012 at the Affiliated Hospital of Nantong University. The mean age of the patients was 34.5 years (range, 23-64 years). TRIzol reagent (Invitrogen; Thermo Fisher Scientific, Inc., Waltham, MA, USA) was used to extract total RNA from the frozen samples. Total RNA was then reverse transcribed using a RevertAid ${ }^{\mathrm{TM}}$ First Strand cDNA Synthesis kit (Fermentas; Thermo Fisher Scientific, Inc.) according to the manufacturer's protocol at $42^{\circ} \mathrm{C}$ for $30 \mathrm{~min}$. For RT-qPCR, the analysis of mRNA levels was performed using SYBR-Green Reagents (Toyobo Life Science, Osaka, Japan) using an iQ5 Multicolor Real-time PCR Detection System, and all mRNA levels were normalized to GAPDH $(n=3)$. The FOXA1 primer sequences were as follows: Forward, 5'-GTT GAAGACTCCAGCCTCCTC-3' and reverse, 5'-CTGCCC AGAACATCATCCCT-3'. GAPDH primers sequences were as follows: Forward, 5'-CGGAGTCAACGGATTTGGTCG TAT-3' and reverse, 5'-AGCCTTCTCCATGGTGGTGAA GAC-3' (Invitrogen; Thermo Fisher Scientific, Inc.). GAPDH mRNA levels were used as an internal control following melt curve analysis (25). Amplification conditions were as follows: Taq activation at $94^{\circ} \mathrm{C}$ for $2 \mathrm{~min} ; 35$ cycles of $94^{\circ} \mathrm{C}$ for $20 \mathrm{sec}$, $58^{\circ} \mathrm{C}$ for $20 \mathrm{sec}$ andelongation at $72^{\circ} \mathrm{C}$ for $30 \mathrm{sec}$.

Immunohistochemical analysis. The $4 \mu \mathrm{m}$ sections were deparaffinized using a graded ethanol series (Xylene 3 times for $3 \mathrm{~min}$, then 100, 95 and 70\%, 3 times for $2 \mathrm{~min}$ each), and $0.3 \%$ hydrogen peroxide was used to block endogenous peroxidase activity at room temperature for $15 \mathrm{~min}$. For antigen retrieval, the sections were placed in $10 \mathrm{mM}$ citrate buffer (pH 6.0) and heated to $121^{\circ} \mathrm{C}$ in an autoclave for $20 \mathrm{~min}$. Goat serum (10\%; Sangon Biotech Co., Ltd., Shanghai, China) was used to block non-specific reactions for $1 \mathrm{~h}$ at room temperature. The sections were then rinsed in phosphate-buffered saline (pH 7.2) and incubated with anti-human FOXA1 antibody (dilution, 1:200; cat. no. ab23738; Abcam, Cambridge, MA, USA) overnight at $4^{\circ} \mathrm{C}$. Then, the sections were incubated with secondary antibody (dilution 1:1,000; cat. no. ab205718; Abcam, Cambridge, MA, USA) at room temperature for $30 \mathrm{~min}$. Sections incubated without antibody were used as the negative control. All slides were processed using the peroxidase anti-peroxidase method (Dako; Agilent Technologies, Inc., Santa Clara, CA, USA). The sections were counterstained with hematoxylin, dehydrated, and coverslips were added subsequent to rinsing with water. The stained sections were observed under a phase contrast microscope (magnification, x400). All immunostained sections were assessed blindly. High-power fields $(n=5)$ for each specimen were randomly selected to assess FOXA1 expression, and nuclear staining was observed under high-power magnification. To determine the mean percentage of immunostained cells, $>500$ cells were counted. The analysis was repeated twice in half of the samples to decrease the likelihood of technical errors; the results of these repeats were similar. If the nuclei were stained $>5 \%$ and the plasma was not, FOXA1 protein expression was considered positive. Staining of $<5 \%$ of the cells was judged as negative, $5-30 \%$ as weak (low expression), $31-70 \%$ as moderate and $>70 \%$ as strong (high expression).

Statistical analysis. SPSS 18.0 software (SPSS, Inc., Chicago, IL, USA) was used to perform statistical analysis. 
A

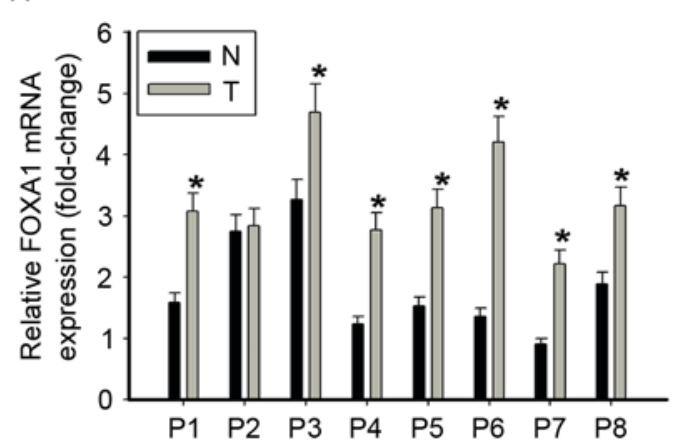

C

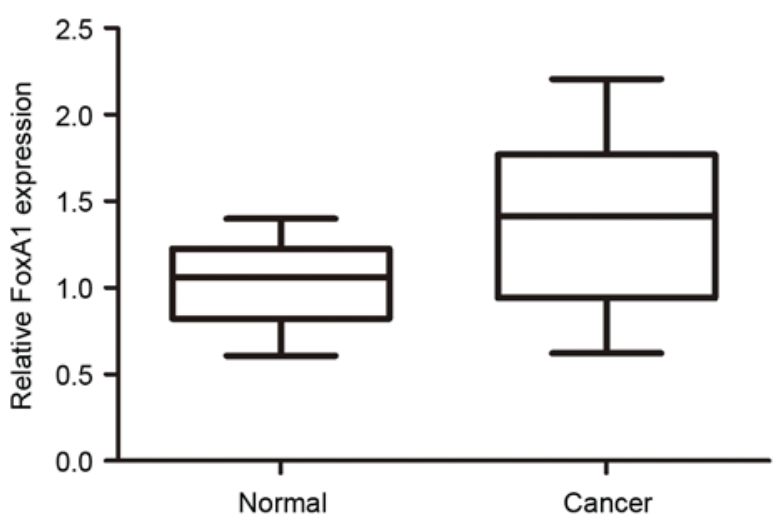

B

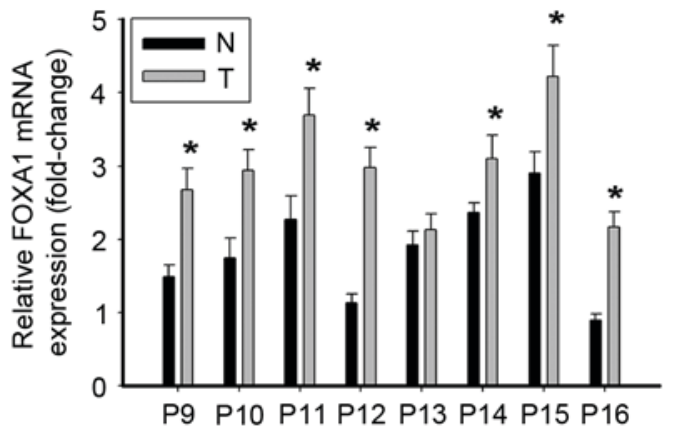

Figure 1. Reverse transcription-quantitative polymerase chain analysis was performed to determinethe expression of FOXA1 mRNA in epithelial ovarian cancer and corresponding normal ovarian tissue. (A) Expression of FOXA1 in 8 paired samples of T and N, including P1-8. (B) Expression of FOXA1 in 8 paired samples of T and N, including P9-16. (C) Mean FOXA1 mRNA expression in cancerous tissuesis increased compared with benign tissues. Error bars indicate the standard error. ${ }^{*} \mathrm{P}<0.05$ vs. N. FOX, Forkhead box; N, normal ovarian tissue; T, ovarian carcinoma tissue; $\mathrm{P}$, pair.

Mean \pm standard deviation was used to represent the results. To compare clinicopathological data, the Fisher's exact test, the $\chi^{2}$ test, and two-sample t-tests were used. Overall survival time was defined as the interval between primary surgery and patient mortality or the final follow-up. Survival curves were plotted using the Kaplan-Meier method. The association between clinical characteristics and survival time in patients with EOC was assessed using the log rank test. $\mathrm{P}<0.05$ was considered to indicate a statistically significant difference.

\section{Results}

FOXA1 mRNA expression differs between EOC and normal ovarian epithelium tissues. In the present study, total RNA was extracted from paired, frozen EOC and normal ovarian epithelium tissues, and subjected to RT-qPCR to measure FOXA1 mRNA expression, normalized to GAPDH mRNA level. FOXA1 expression was increased in EOC specimens compared with the corresponding normal tissue (Fig. 1A and B). The mean relative expression of FOXA1 mRNA in EOC and the corresponding benign ovarian tumor surface epithelium tissues was $1.389 \pm 0.1225$ and $1.029 \pm 0.0626$, respectively, indicating a significant difference $(\mathrm{P}=0.014$; Fig. 1C).

Expression of FOXA1 protein in epithelial ovarian tumor and non-cancerous tissues. To confirm the increased expression of FOXA1 in EOC tissues compared with non-cancerous tissues, immunohistochemical staining was performed. FOXA1 expression was primarily observed in the nuclei and not inthe cytoplasm (Fig. 2). As revealed by immunohistochemical analysis, FOXA1 expression was upregulated in EOC. Only $10.0 \%$ of the normal ovarian tissues demonstrated moderate or strong positive expression of FOXA1: 20.8 and $73.6 \%$ of the benign ovarian tumor surface epithelium tissues and EOC specimens, respectively, revealed moderate or strong positive expression of FOXA1 (Table I). Furthermore, the percentage of FOXA1 positive cells increased with an increasing WHO grade in EOC tissue: $84.8 \%$ of high-grade (III-IV) and $65.6 \%$ of low-grade (I-II) OC tissues exhibited moderate or strong expression of FOXA1 (Table I). Therefore, the total expression of FOXA1 in EOC tissues was significantly increased compared with non-cancerous tissues $(\mathrm{P}<0.001)$, and increased with increasing tumor grade $(\mathrm{P}=0.024)$ (Table II). The results indicated that the mean value of FOXA1 immunoreactivity in benign ovarian tumor surface epithelium tissues was not significantly differentcompared with normal ovarian tissues $(\mathrm{P}=0.450)$.

FOXA1 expression in EOC is associated with a reduced survival time. To determine the association between FOXA1 expression and clinicopathological characteristics in EOC, the clinical data of 110 patients with EOC were analyzed (Table II). Increased expression of FOXA1 in EOC was significantly associated with the tumor WHO grade $(\mathrm{P}=0.024)$ and differentiation status $(\mathrm{P}=0.003)$. There was no significant 

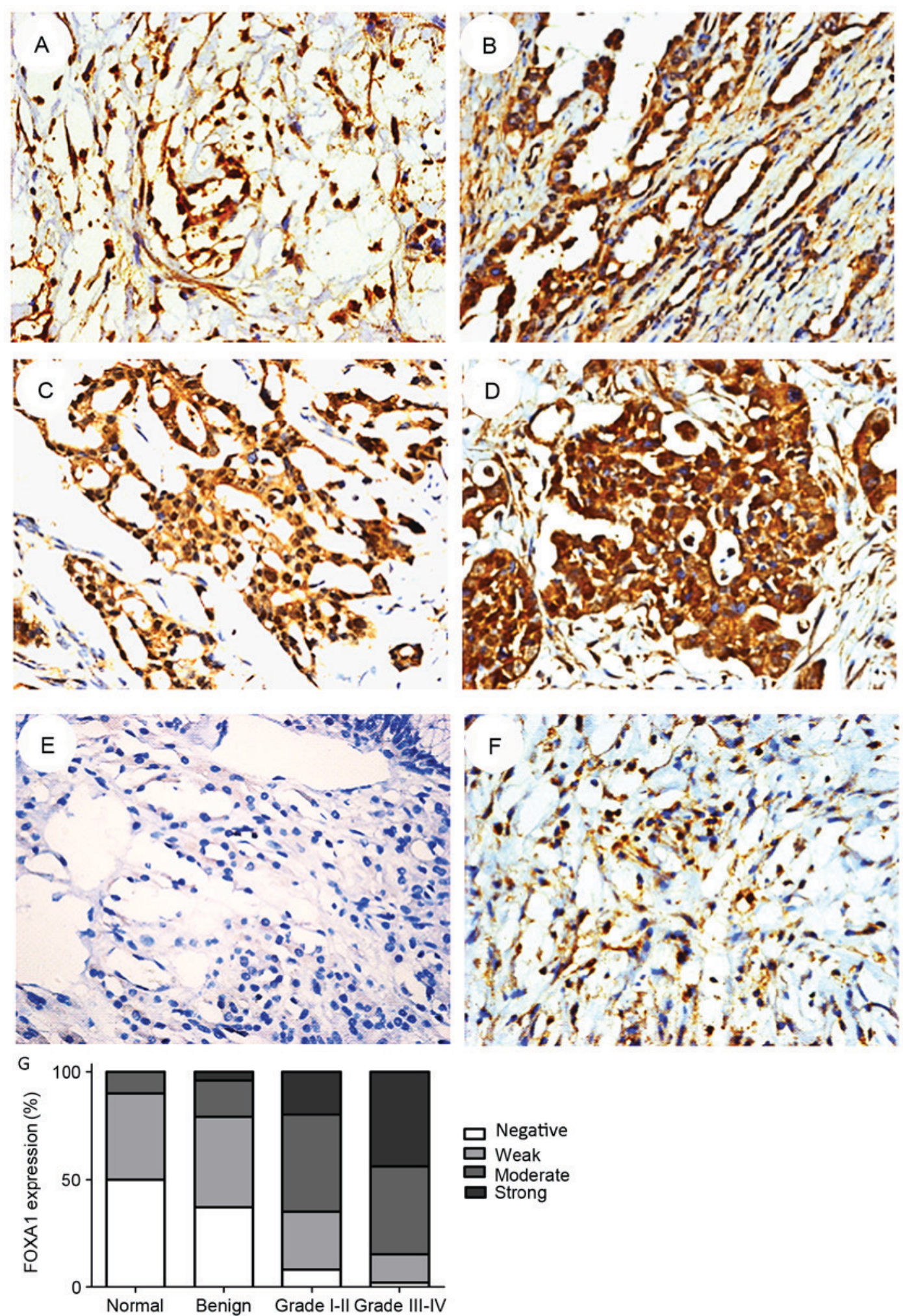

Figure 2. Immunohistochemical analysis of FOXA1 expression in non-malignant ovarian and EOC tissue samples. FOXA1 immunoreactivity was detected in EOC; staining was predominantly identified in the nuclei (magnification, $x 400$ ). Representative images of (A) stage I, (B) stage II, (C) stage III (D) stage IVEOC. (E) Representative image of FOXA1 expression innormal ovarian surface epithelia. (F) Representative image of FOXA1 expression in benign ovarian tumor surface epithelia. (G) FOXA1 expression was increased in grade III-IV EOC tissues compared with grade I-II EOC and non-malignant ovarian tissues. FOX, Forkhead box; EOC, epithelial ovarian cancer.

association between FOXA1 expression and age, histological subtype, tumor diameter or location. Furthermore, increased FOXA1 expression in non-cancerous epithelial ovarian tissues was not significantly associated with the clinical characteristics of EOC (data not shown). However, the overall survival time of the patients in the FOXA1 low expression and high expression groups differed significantly ( $\mathrm{P}=0.013)$ (Fig. 3). The patients with low expression of FOXA1 exhibited increased overall 
Table I. Expression of FOXA1 in normal ovarian and ovarian cancer samples of different grades.

FOXA1 expression

Group Cases, $\mathrm{n}$ Negative Weak Moderate Strong

\begin{tabular}{llrrrr}
\hline Normal & 10 & 5 & 4 & 1 & 0 \\
Benign & 24 & 9 & 10 & 4 & 1 \\
Grade I/II & 64 & 5 & 17 & 29 & 13 \\
Grade III/IV & 46 & 1 & 6 & 19 & 20
\end{tabular}

FOXA1, Forkhead box A1.

survival time compared with those with high expression of FOXA1, indicating that FOXA1 expression may exhibit prognostic value for patients with EOC.

\section{Discussion}

OC is the second most common type of gynecological cancer worldwide, and a major cause of cancer-associated mortality in women (2). Unlike other reproductive malignancies, including prostate cancer and breast carcinoma, EOC lacks an established biomarker for screening. Identifying biomarkers for EOC may reveal novel therapeutic targets and provide a potential screening test. The present study assessed the expression of FOXA1 in EOC to determine its diagnostic or prognostic value.

The FOX proteins are a large family that is divided into 17 subclasses (A-Q) according to the amino acid sequence of their conserved Forkhead domains (26). They are associated with embryonic development, cell cycle regulation, cellular proliferation, transformation, immune regulation, differentiation, longevity and multiple other biological processes; mutation and expression abnormalities to proteins of this family may be associated with developmental abnormalities, metabolic diseases and tumor occurrence $(27,28)$. FOXA1, a member of the FOXA subclass of FOX transcription factors, mediates the nuclear steroid receptor signaling pathwayby regulating androgen receptor and estrogen receptor activity (26). FOXA1 serves a major function in modulating nuclear steroid receptor activity in breast and prostate cancer, and it has been suggested that FOXA1 may be associated with pro-tumorigenic phenotypes (29). FOXA1 is necessary for the estrogen signaling pathway to function in breast cancer cells, and its expression has been associated with improved prognosis in patients with breast cancer $(19,20)$. It has also been suggested that FOXA1 influences AR binding to chromatin in androgen-dependent and androgen-independent prostate cancer $(21,22)$. As EOC is also hormone-dependent, this prompted the assessment of FOXA1 expression in EOC.

The present study demonstrated that the expression of FOXA1 mRNA and protein in EOC tissues was significantly increased compared with that in non-malignant tissues. The results of immunohistochemical analysis were consistent with those of RT-qPCR analysis, which suggested that the expression of FOXA1 may serve an important function in
Table II. FOXA1 expression and clinicopathological parameters in 110 ovarian cancer specimens, including a comparison to normal and benign specimens.

\begin{tabular}{|c|c|c|c|c|}
\hline \multirow[b]{2}{*}{ Characteristic } & \multirow[b]{2}{*}{ Cases, $\mathrm{n}$} & \multicolumn{2}{|c|}{$\begin{array}{l}\text { FOXA1 } \\
\text { expression, } \mathrm{n}\end{array}$} & \multirow[b]{2}{*}{ P-value } \\
\hline & & Low & High & \\
\hline Age, years & & & & 0.424 \\
\hline$\leq 55$ & 54 & 21 & 33 & \\
\hline$>55$ & 56 & 26 & 30 & \\
\hline Status & & & & $<0.001$ \\
\hline Normal & 10 & 9 & 1 & \\
\hline Benign & 24 & 19 & 5 & \\
\hline Invasive & 110 & 29 & 81 & \\
\hline Clinical stage & & & & 0.024 \\
\hline I-II & 64 & 22 & 42 & \\
\hline III-IV & 46 & 7 & 39 & \\
\hline Histological subtype & & & & 0.768 \\
\hline $\begin{array}{l}\text { Serous papillary } \\
\text { adenocarcinoma }\end{array}$ & 80 & 36 & 44 & \\
\hline $\begin{array}{l}\text { Endometrioid } \\
\text { adenocarcinoma }\end{array}$ & 12 & 3 & 9 & \\
\hline Clear cell carcinoma & 9 & 4 & 5 & \\
\hline Mucinous carcinoma & 5 & 2 & 3 & \\
\hline $\begin{array}{l}\text { Transitional cell } \\
\text { carcinoma }\end{array}$ & 4 & 2 & 2 & \\
\hline Differentiation & & & & 0.003 \\
\hline Good & 48 & 30 & 18 & \\
\hline Moderate & 26 & 12 & 14 & \\
\hline Poor & 36 & 9 & 27 & \\
\hline Tumor diameter, $\mathrm{cm}$ & & & & 0.179 \\
\hline$\leq 5$ & 49 & 28 & 21 & \\
\hline$>5$ & 61 & 27 & 34 & \\
\hline Tumor location & & & & 0.061 \\
\hline Unilateral & 59 & 36 & 23 & \\
\hline Bilateral & 51 & 22 & 29 & \\
\hline
\end{tabular}

FOXA1, Forkhead box A1.

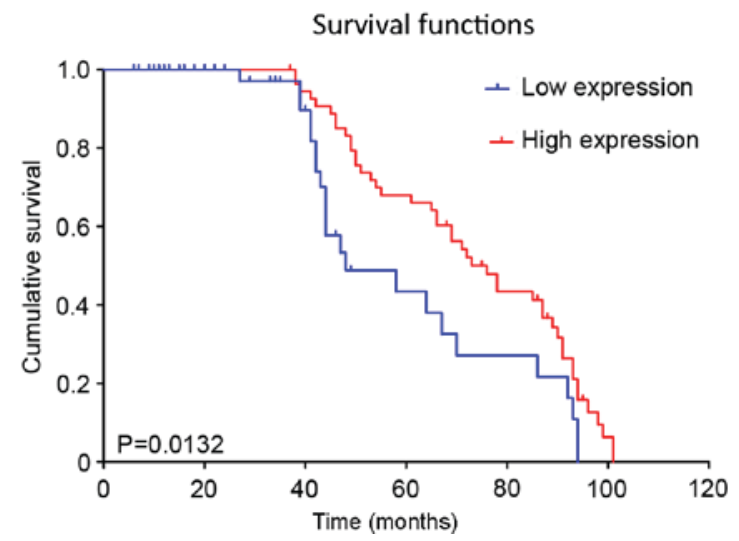

Figure 3. Kaplan-Meier survival curves for patients with epithelial ovarian cancer stratified by the high or low expression of Forkhead boxA1. 
EOC tumorigenesis. The present study also revealed that the alteration to FOXA1 expression was associated with the WHO grade of EOC, clinicopathological characteristics and patient survival time. The increased expression of FOXA1 was associated with an increased WHO grade, poor differentiation and reduced overall survival time irrespective of age, histological type, tumor size or location. The results of the present study suggest that FOXA1 expressionmaypredict survival timein patients with OC.FOXA1may represent a novel prognostic factor and potential therapeutic target for patients with $\mathrm{OC}$ as an important transcription factor in EOCand other types of malignancy.

In conclusion, the present study revealed that FOXA1 was overexpressed in EOC tissues compared with normal tissue. The level of FOXA1 expression was associated with the tumor grade, differentiation status and prognosis. The results of the present study indicated that FOXA1 may serve an important function in EOC and may be a potential therapeutic target and prognostic marker. However, the function of FOXA1 and its role in tumorigenesis in EOC have yet to be fully characterized, and require additional study.

\section{References}

1. Auersperg N, Wong AS, Choi KC, Kang SK and Leung PC: Ovarian surface epithelium: Biology, endocrinology, and pathology. Endocr Rev 22: 255-288, 2001.

2. Siegel R, Ma J, Zou Z and Jemal A: Cancer statistics, 2014. CA Cancer J Clin 64: 9-29, 2014

3. Jemal A, Siegel R, Xu J and Ward E: Cancer statistics, 2010. CA Cancer J Clin 60: 277-300, 2010.

4. Ozols RF: Treatment goals in OC. Int J Gynecol Cancer 15 (Suppl 1): S3-S11, 2005.

5. Schink JC: Current initial therapy of stage III and IV OC: challenges for managed care. Semin Oncol 26 (1 Suppl 1): S2-S7, 1999.

6. Lin L, Miller CT, Contreras JI, Prescott MS, Dagenais SL, Wu R, Yee J, Orringer MB, Misek DE, Hanash SM, et al: The hepatocyte nuclear factor 3 alpha gene, HNF3alpha (FOXA1), on chromosome band 14q13 is amplified and overexpressed in esophageal and lung adenocarcinomas. Cancer Res 62: 5273-5279, 2002.

7. Wolf I, Bose S, Williamson EA, Miller CW, Karlan BY and Koeffler HP: FOXA1: Growth inhibitor and a favorable prognostic factor in human breast cancer. Int J Cancer 120: 1013-1022, 2007.

8. Lantz KA and Kaestner KH: Winged-helix transcription factors and pancreatic development. Clin Sci (Lond) 108: 195-204, 2005

9. Costa RH, Grayson DR and Darnell JE Jr: Multiple hepatocyte-enriched nuclear factors function in the regulation of transthyretin and alpha 1-antitrypsin genes. Mol Cell Biol 9: 1415-1425, 1989.

10. Lai E, Prezioso VR, Smith E, Litvin O, Costa RH, Darnell JE Jr: HNF-3A, a hepatocyte-enriched transcription factor of novel structure is regulated transcriptionally. Genes Dev 4: 1427-1436, 1990.
11. Carroll JS and Brown M: Estrogen receptor target gene: An evolving concept. Mol Endocrinol 20: 1707-1714, 2006.

12. Friedman JR and Kaestner KH: The Foxa family of transcription factors in development and metabolism. Cell Mol Life Sci 63: 2317-2328, 2006.

13. Lee CS, Friedman JR, Fulmer JT and Kaestner KH: The initiation of liver development is dependent on Foxa transcription factors. Nature 435: 944-947, 2005.

14. Besnard V, Wert SE, Hull WM and Whitsett JA: Immunohistochemical localization of Foxa1 and Foxa2 in mouse embryos and adult tissues. Gene Expr Patterns 5: 193-208, 2004.

15. Cirillo LA, Lin FR, Cuesta I, Friedman D, Jarnik M and Zaret KS: Opening of compacted chromatin by early developmental transcription factors HNF3 (FoxA) and GATA-4. Mol Cell 9: 279-289, 2002.

16. Gualdi R, Bossard P, Zheng M, Hamada Y, Coleman JR and Zaret KS: Hepatic specification of the gut endoderm in vitro: Cell signaling and transcriptional control. Genes Dev 10: 1670-1682, 1996.

17. Nakshatri $\mathrm{H}$ and Badve S: FOXA1 as a therapeutic target for breast cancer. Expert Opin Ther Targets 11: 507-514, 2007.

18. Nucera C, Eeckhoute J, Finn S, Carroll JS, Ligon AH, Priolo C, Fadda G, Toner M, Sheils O, Attard M, et al: FOXA1 is a potential oncogene in anaplastic thyroid carcinoma. Clin Cancer Res 15: 3680-3689, 2009.

19. Badve S, Turbin D, Thorat MA, Morimiya A, Nielsen TO, Perou CM, Dunn S, Huntsman DG and Nakshatri H: FOXA1 expression in breast cancer-correlation with luminal subtype A and survival. Clin Cancer Res 13: 4415-4421, 2007.

20. Thorat MA, Marchio C, Morimiya A, Savage K, Nakshatri H, Reis-Filho JS and Badve S: Forkhead box A1 expression in breast cancer is associated with luminal subtype and good prognosis. J Clin Pathol 61: 327-332, 2008.

21. Lupien M, Eeckhoute J, Meyer CA, Wang Q, Zhang Y, Li W, Carroll JS, Liu XS and Brown M: FoxA1 translates epigenetic signatures into enhancer-driven lineage-specific transcription. Cell 132: 958-970, 2008.

22. Wang Q,LiW,Zhang Y, Yuan X,XuK, Yu J,ChenZ, BeroukhimR, Wang $\mathrm{H}$, Lupien $\mathrm{M}$, et al: Androgen receptor regulates a distinct transcription program in androgen-independent prostate cancer. Cell 138: 245-256, 2009.

23. Zeppernick F and Meinhold-Heerlein I: The new FIGO staging system for ovarian, fallopian tube and primary peritoneal cancer. Arch Gynecol Obstet 290: 839-842, 2014.

24. Hauptmann S, du Bois A, Meinhold-Herlein I, Pfisterer J and Avril S: Histological grading of epithelial ovarian cancer. Review and recommendation. Pathologe 35: 497-503, 2014.

25. Vinayagamoorthy T, Maryanski D, Vinayagamoorthy D, Hay KS, Yo J, Carter M and Wiegel J: Improved internal control for molecular diagnosis assays. MethodsX 2: 159-164, 2015.

26. Kaestner KH, Knochel W and Martinez DE: Unified nomenclature for the winged helix/forkhead transcription factors. Genes Dev 14: 142-146, 2000.

27. Lehmann OJ, Sowden JC, Carlsson P, Jordan T and Bhattacharya SS: Fox's in development and disease. Trend Genet 19: 339-344, 2003.

28. Tan Y, Raychaudhuri P and Costa RH: Chk2 mediates stabilization of the FoxM1 transcription factor to stimulate expression of DNA repair genes. Mol Cell Biol 27: 1007-1016, 2007.

29. Robinson JL and Carroll JS: FoxA1 is a key mediator of hormonal response in breast and prostate cancer. Front Endocrinol (Lausanne) 3: 68, 2012. 